\title{
Establishing a Hardware-in-the-Loop Research Environment with Hybrid Energy Storage System
}

\author{
Sebastian Kochanneck ${ }^{1 *}$, Ingo Mauser ${ }^{1}$, Bernd Bohnet ${ }^{2}$, Sebastian Hubschneider ${ }^{3}$, \\ Hartmut Schmeck ${ }^{1}$, Michael Braun ${ }^{2}$ and Thomas Leibfried ${ }^{3}$ \\ Karlsruhe Institute of Technology, Germany; ${ }^{1}$ Institute AIFB, ${ }^{2}$ Institute ETI, ${ }^{3}$ Institute IEH; \\ *Telephone: +49 72160845712 (main contact); Email: [firstname].[lastname]@kit.edu
}

\begin{abstract}
The KIT Energy Smart Home Lab is a smart residential building comprising building automation, metering systems, sensors, intelligent appliances, heating, ventilation, and air-conditioning equipment, distributed generation, and energy storage systems. Currently, the lab is extended by a hybrid energy storage system and a linear voltage amplifier for real-time simulations, to facilitate fully functional power hardware-in-the-loop simulations and evaluations. This paper presents the setup of the lab, the hardwarein-the loop research environment, and first measurements when using a simulated artificial mains network.
\end{abstract}

\section{INTRODUCTION AND Motivation}

The electrical energy systems are in a state of transition from the traditional power grid and large power plants to a smart grid and distributed generation (DG). The German Energiewende for instance is driven by political, economical, and ecological considerations, leading to a paradigm shift from "generation follows demand" to "demand follows generation". In particular, low voltage (LV) systems are in a transition from being the passive demand side to becoming an active part of the grid, reacting on intermittent generation from renewable energy sources (RES). Therefore, distribution grids will become more dynamic and unstable.

To assess mechanisms and technologies that help coping with DG without expensive expansions of the grid, detailed simulations of typical grid structures and entities are inevitable. Therefore, detailed bottom-up models and simulations of distribution grids as well as (power) hardware-in-the-loop (HIL/PHIL) evaluations are proposed to demonstrate the effects of smart buildings with building energy management systems (BEMS) and energy storage systems (ESS). HIL simulations allow for an assessment of real components under variable operating conditions and their evaluation in critical situations and under fault conditions.

Sect. II presents the context, challenges and research questions of this paper. After an overview of our lab is given in Sect. III, the building management, a BEMS and a hybrid energy storage control system (HESCOS), is presented in Sect. IV. Before the paper is concluded by an outlook, first results of HIL evaluations are presented in Sect. V.

\section{RESEARCH QUESTIONS AND APPROACH}

The HIL research environment in the Energy Smart Home $L a b^{1}$ at the Karlsruhe Institute of Technology (KIT) has been

\footnotetext{
${ }^{1}$ http://www.aifb.kit.edu/web/Energy_Smart_Home_Lab
}

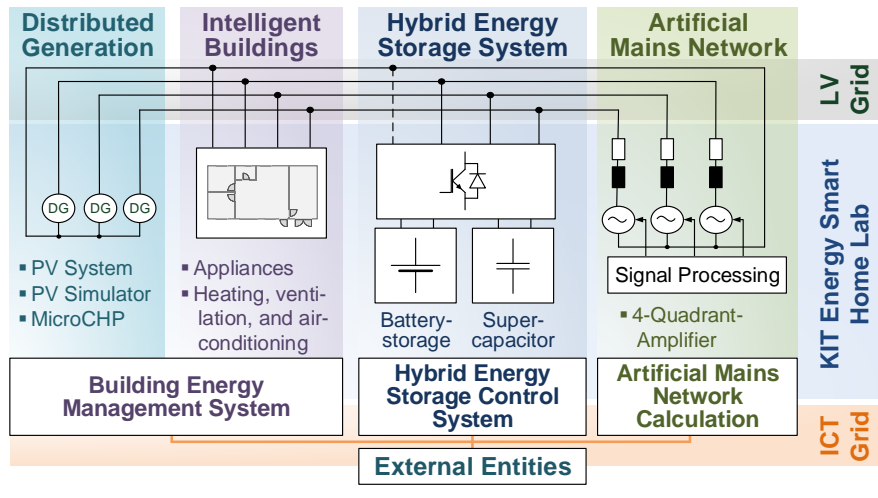

Figure 1: System approach for a soft- and hardware test bench and power hardware-in-the-loop evaluations

set up to address questions in the context of grid stability in LV grids comprising many inverters and volatile generation as well as the distributed provision of ancillary grid services.

\section{A. General Challenges}

The ESHL is used to develop and orchestrate technologies and solutions for low voltage distribution grids. Solutions for several identified challenges in distribution grids that can be separated into two groups will be developed: Firstly, there are stability challenges and congestions caused by volatile generation and a lack of reactive power, both decreasing the voltage stability of the grid. Furthermore, the substitution of rotating generators by inverters is decreasing the amount of available short-circuit power within the grid. Within this context, the usage of ESS has to be considered and changed from uncoordinated to smart control that supports the grids. The second group of challenges has a long term planning and economical background: Due to the currently rather small number of sensors within distribution grids the observability of those grids is challenging and has to be improved by new methods. Additionally, a future energy and grid planning can strongly benefit from precise power flow predictions.

\section{B. Stability of Distribution Grids}

As pointed out in the motivation, the amount of DG and their volatility is challenging the voltage stability and can cause congestion issues. Many devices in 3-phase energy systems are connected to the grid using only a single phase, causing a 
decrease in power quality because of phase imbalances as well as harmonics. Hence, voltage control, phase balance, and congestion handling techniques have to be developed and applied to LV systems to guarantee a stable grid operation.

Furthermore, PV and ESS are connected to the grid using inverters. Due to the fact that inverters can only provide a fraction of the short circuit power of a generator or transformer, the future grid is likely to face problems of fault clearance when having a lack of short circuit power provision. Hence, there is a need for cost efficient techniques providing short circuit power to enable a safe system operation with faultride-through capabilities. These problems are intensified by the uncoordinated usage of ESS, i. e., highly dynamic systems with noticeable power rating. Thus, ESS have to be controlled in a better way that reduces stability issues while still allowing for an economical efficient usage.

However, inverters have a high potential for grid control applications: Due to the capability of controlled feed-in of reactive power the voltage level of LV grids can be influenced strongly [1], [2]. Again, there is a lack of tested control mechanisms in real environments, not least because of regulatory restrictions on energy grids.

To cope with all these challenges in LV systems, control and optimization systems affecting different components have to cooperate and support the provision of services to stabilize the system in terms of power balancing in critical situations, power quality increase, distributed provision of ancillary services, incentive based load control, and a more grid-supporting integration of energy storage. All these tasks have dependencies with each other and cannot be considered alone.

To develop suitable and practical solutions that may be applied to the real world successfully, a realistic test bench is needed. Obviously, some scenarios can not be assessed in an inhabited residential LV system for safety, regulatory, and economical reasons. Therefore, a approach of a combined hardand software test bench system for smart LV grid technologies is presented.

\section{General Approaches}

In order to assess technologies for the stated problems and challenges, a test bench integrating detailed bottom-up simulated buildings and devices as well as real electrical equipment and hardware of LV systems is presented, leading to an overall (power) hardware-in-the-loop solution. This allows for a long time support during the research and development on new technologies.

a) Simulation of Buildings: Residential buildings in a LV grid have already been simulated in a bottom-up manner [3]: Utilizing the simulation capabilities of a BEMS, a generic building model has been parametrized to resemble different kinds of residential buildings by using statistical data and timeof-use surveys. The simulated buildings have been connected to a static, 1-phase LV grid model; some of them equipped with DG. This allows for static analyses of the effects of intelligent buildings within grids [4]. b) Simulation of LV Grids: While the so far described simulations focus on static scenarios of LV systems, dynamic simulations are needed for evaluation of fast acting entities: Static assessments allow to use more simple electric models, e.g. 1-phase instead of 3-phase load flow calculations, facilitating parameter studies on effects with high time constraints like virtual power plants. However, these simplifications cannot be made for dynamic or transient evaluations, like an inverter's feedback to the grid. To deal with the high number of calculations per voltage period (high temporal resolution), the previously modeled LV grid is abstracted into one simplified equivalent circuit diagram in a first attempt. Here, remote nodes from the feed, i. e. critical weak connection points, are in focus. These nodes can be identified using the static evaluations presented above.

c) Hardware-in-the-Loop: The approach to evaluate (parts of) real components within a simulated environment is called hardware-in-the-loop (HIL). Hereby, the complexity of the development of cyber-physical systems can be reduced by adding repeatable degrees of freedom to the design phase of the system. This allows for the reproduction of adjustable system constraints, as well as critical and extreme situations, in a laboratory environment. A real-time capable simulator recreates the expected system environment (the simulated LV grid) and provides interfaces to integrate the system to be evaluated. The information exchange between the system and the simulator is realized via sensor-actuator-couplings building a closed-loop system. Within a HIL environment the coupling is realized via low-level signals, like sensor data and control signals [5], [6].

To realize a HIL approach within the laboratory (see Sect. III) the simulation approach (see above) is extended by the possibility to replace a simulated building with the lab. Measurements, e.g. active powervoltages, or harmonics, are fed into the simulation of a LV system. The states of the simulated power grid are inputs for grid control and BEMS. The control signals of these systems are sent back to the lab, where the system's reaction to these signals can be evaluated.

d) Power Hardware-in-the-Loop: In principal, power hardware-in-the-loop (PHIL) works just like a HIL system, only that the signals that are being exchanged between the simulator and the hardware are large scale signals, i. e. energy grid voltages and resulting currents.

Hence, an actor is needed that can provide the behavior of a grid connection point. The properties of this point are determined by a LV grid simulation (see above) in hard realtime that is influenced by the behavior of the connected physical system. To build such an artificial mains, a 4-quadrant amplifier, an analog driven linear voltage amplifier, can be utilized. It delivers the consumed power of the ESHL, as well as it is able to handle their produced electrical power. Afterwards, flowing currents are measured and fed back to a real-time simulation in order to represent the behavior of the real hardware for the next grid state calculation. For dynamic simulations the loop times should not exceed $\sim 20 \mu$ s. 


\section{Integrated Approach}

In Fig. 1, the big picture of the LV test system laboratory is depicted. The main idea is to integrate all aspects of LV systems into one lab environment allowing for conventional, HIL, and PHIL evaluations and simulations. DG, appliances, and hybrid ESS are integrated into one LV system that can be fed by an artificial mains network. The hardware is controlled by different, interacting control systems: a building energy management system and a hybrid energy storage control system that can both interact with external entities. Because a full scale LV system containing a multitude of households, DG, electric vehicles, and utility equipment can not be built, the approach is to set up just one entity of a LV system in real hardware: The KIT Energy Smart Home Lab. The missing components have to be simulated while using an artificial mains network calculation. Both components are coupled to (P)HIL simulations and evaluations by using the lab's sensors as well as external actors, like a 4-quadrant amplifier.

\section{E. Similar Research Environments}

A similar environment where energy related technologies have been integrated into an in-use commercial building is presented in [7]. Electrical and thermal components are integrated and monitored by various sensors. The gathered energy data is used for energy management and user interaction via a visualization. A large scale research environment to help answer questions of the "Energiewende" is presented in [8]. It contains a PHIL environment, an energy grid analysis laboratory, as well as a lab for visualization and control. Here, especially the large data flows within future energy systems are addressed and the ESHL will be interconnected to it. A HIL environment for evaluation of ICT controllers and protection equipment with focus on network integration is presented in [9]. An overview of more similar lab environments is given in [7]. Nevertheless, no one has presented PHIL evaluation of LV entities, like an intelligent building.

\section{KIT ENergy SMart Home Lab ENVIRONMENT}

The KIT Energy Smart Home Lab (ESHL) is a residential building containing a $60 \mathrm{~m}^{2}$ two-bedroom apartment and a $20 \mathrm{~m}^{2}$ technical room (see Fig. 2). All relevant installed devices are listed in Tab. I.

\section{A. Appliances}

Demand response techniques using appliances have proven their potential, e. g., in [10], [11]. Within the ESHL the washing machine, tumble dryer and dishwasher are integrated into the BEMS for load flexibility. After a user has specified a temporal degree of freedom, i. e., the time when the appliance must have finished its program, the BEMS determines the appliance's best possible start time with respect to given goals.

\section{B. Distributed Generation}

To evaluate technologies for the integration of the volatile and difficult to predict PV, a PV system with a total power of $4.7 \mathrm{~kW}_{\text {peak }}$ is installed on the lab's roof. It is connected to a

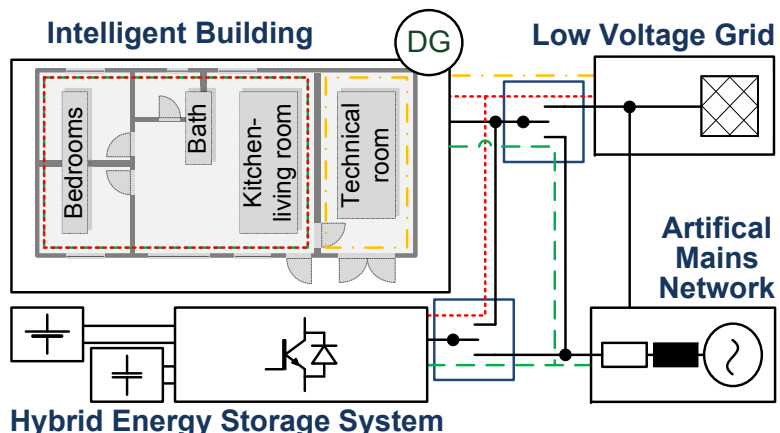

Figure 2: Energy Smart Home Lab: electrical installation

3-phase inverter with a maximum output power of $10.0 \mathrm{kVA}$. The inverter's parameters, e.g. its power factor or active power restrictions, can be dynamically set by the BEMS.

Besides the PV a gas driven microCHP with a thermal output power of $12.5 \mathrm{~kW}$ and $5.5 \mathrm{~kW}$ electric output power has been installed. The heat is used within the ESHL for domestic hot water supply and for space heating. The heat is cached in a hot water tank of 7501 . This storage is utilized for a flexible, electricity driven operation of the microCHP. Its operation is controlled and optimized by the BEMS described in Sect. IV.

For the usage of the surplus energy generated by the microCHP or the PV a $9 \mathrm{~kW}$ electrical insert heating element is installed in the hot water tank. The nominal power is determined by the output power of the microCHP, the PV, and the house's baseload. Nevertheless, an insert heating element with controllable power output provides additional flexibility for the optimization [11].

It is important for evaluations of a microgrid to use similar PV generation profiles for the generation real and simulated PV systems.

To feed the ESHL with generated or recorded PV profiles, a controllable DC power supply with a maximum output power of $3 \mathrm{~kW}$ is installed, representing a PV simulator for the ESHL that is also connected to the PV inverter.

\section{Hybrid Electrical Energy Storage System}

Stationary ESS enable additional optimization possibilities. For evaluation purposes a hybrid storage system (HSS), combining supercapacitors, i. e., electric double layer capacitors (EDLC), and batteries is built and integrated into the ESHL.

Tab. II shows the volumetric power and energy density of the proposed storage system. Regarding these values, EDLC are a perfect addition for batteries: The high energy density of batteries enables an optimized usage of energy generated by the installed DG, whereas EDLCs feature a very high power density, so they are ideal to smooth high power gradients.

These could have multiple reasons, like fluctuating energy delivery from PV systems during cloudy days or fast switch on of heavy loads. Especially in weak grids, these peaks may lead to unwanted sudden changes in grid voltage (flicker). Other important fields with high power demands during short times are substitution of rotating mass and fault ride through 
Table I: Energy Smart Home Lab: technical data of the installed devices and systems

\begin{tabular}{|c|c|c|}
\hline \multicolumn{3}{|l|}{ Distributed Generation } \\
\hline PV panels & 24x Sovello SV-T-195 & $4.7 \mathrm{~kW}_{\text {peak }}$ \\
\hline PV inverter & SMA Sunny Tripower STP 10000TL-10 & $10.0 \mathrm{kVA}$ (3-phase) \\
\hline PV simulator & ET System LAB/SMS3100 & $3.0 \mathrm{~kW}$ \\
\hline MicroCHP & SenerTec Dachs G5.5 standard & $5.5 \mathrm{~kW}_{\text {electrical }}, 12.5 \mathrm{~kW}_{\text {thermal }}$ \\
\hline \multicolumn{3}{|l|}{ Home Appliances } \\
\hline Appliances & \multicolumn{2}{|c|}{$\begin{array}{l}\text { Miele: coffee machine, dishwasher, dryer, hob, oven, washing machine; } \\
\text { Liebherr: deep freezer, refrigerator; other: microwave, water kettle, toaster }\end{array}$} \\
\hline Appliance gateway & Miele XGW 2000 & Communication BEMS \& appliances \\
\hline \multicolumn{3}{|c|}{ Heating and Air-conditioning System } \\
\hline Climate controller & Kieback \& Peter BMR410 and FBU410 & Modbus gateway \\
\hline Hot water storage tank & SenerTec SE 750 & 750 liters $(\approx 25 \mathrm{kWh}$ for $\Delta \theta=30 \mathrm{~K})$ \\
\hline Insert heating element & Eltra $2 N P 5635-290$ & $9 \mathrm{~kW}$ \\
\hline Air-conditioning inverter & Mitsubishi PUHZ-RP6OVHA4 & $6 \mathrm{~kW}$ cooling capacity \\
\hline Chilled water storage tank & Custom-made & 200 liters \\
\hline Phase change material & DeltaSystems DELTA-COOL 24 & Melting temperature: $22-28^{\circ} \mathrm{C}$ \\
\hline \multicolumn{3}{|c|}{ Hybrid Electrical Energy Storage System } \\
\hline Battery & 12x Hoppecke power.com HC122000 & $7.920 \mathrm{kWh}$ (three hour discharge) \\
\hline EDLC & 5x SPS MCE0010C0-0090ROTBA & $40.32 \mathrm{kWs}$ (per module) \\
\hline \multicolumn{3}{|c|}{ Metering Systems and Sensors } \\
\hline Electrical metering system & WAGO 750-8204 & WAGO-I/O-SYSTEM 750 controller \\
\hline Electrical meters & WAGO 750-494 and 750-495 & 3-phase and 4-phase electrical meters \\
\hline Phasor measurement unit & Electric Data Recorder & 3-phase power quality, $25 \mathrm{kHz}$ \\
\hline Thermal metering system & Lingg \& Janke eibSOLO & KNX gateway \\
\hline Thermal meters & Kamstrup MULTICAL 601 & KNX heating and cooling meters \\
\hline VOC sensors & AppliedSensor AS-MLV-P & USB VOC sensors \\
\hline Radio beacons & blukii SmartSensor / S & Bluetooth Low Energy sensor beacons \\
\hline \multicolumn{3}{|l|}{ Artificial Mains Network } \\
\hline Grid switching box & Custom-Made & interruption-free supply system switching \\
\hline
\end{tabular}

Table II: Characteristics of used storage technologies

\begin{tabular}{lcc}
\hline & Energy Density Wh/l & Power Density W/l \\
\hline Battery & 71.5 & 71 \\
EDLC & 1.4 & 1013 \\
\hline
\end{tabular}

conditions, so the HSS will enable provision of advanced ancillary services maintaining power quality in the LV grid.

The final storage will be composed of lead-acid batteries with an energy content of $\sim 8 \mathrm{kWh}$ and up to five EDLC-modules enabling a peak power of $22 \mathrm{~kW}$ for several seconds.

The HSS is interfaced to the grid with a custom made power converter. Its control system (Sect. IV) allows a flexible and robust grid current injection even during faults.

\section{Sensors}

Within the ESHL, a modular system for electrical metering and building automation is used. Programmable logic controllers are used to couple the low level meters and actors with the control and optimization systems. Every electrical device within the smart home is monitored secondly and meters for power quality are monitoring the building's grid connection point. For this purpose the Electrical Data Recorder [12] provides high rate voltage time series. The building automation allows for remote control of lights and rolling shutters. The heat and cold flow through each room is monitored by separate thermal meters and dew-point sensors. In addition to the metering systems, volatile organic compounds (VOC) sensors measure the air quality within the lab. Combined with a Bluetooth Low Energy presence detection system these values are used to detect occupancy and control heating and cooling.

\section{E. 4-Quadrant Amplifier and Hardware Integration}

To evaluate the behavior of the installed systems under real but adjustable conditions, a 3-phase 4-quadrant amplifier that recreates an artificial mains is used. The ESHL is fed by up to $10.0 \mathrm{kVA}$ per phase with an adjustable voltage. It is depending on the consumed power and the defined line impedance. In times of high DG feed-in, variable heating resistors included in the system absorb up to $5.0 \mathrm{~kW}$ power per phase.

The ESHL's electric installation is separated into three grids (see Fig. 2): a smart home grid where the appliances and DG are connected (red), a plant room grid for supply of sensors and simulators (orange), and a storage grid for the hybrid storage system (green). Whereas the plant room grid is always connected to a stiff grid generated by a distribution transformer, the smart home and storage grid can be connected individually either to the stiff grid or to the artificial mains. This allows for the evaluation of utility equipment under fault conditions that would endanger the components of the smart home. A switch has been installed to allow interruption-free automated switching of the smart home / battery grid from static to artificial mains (results see Sect. VI). 


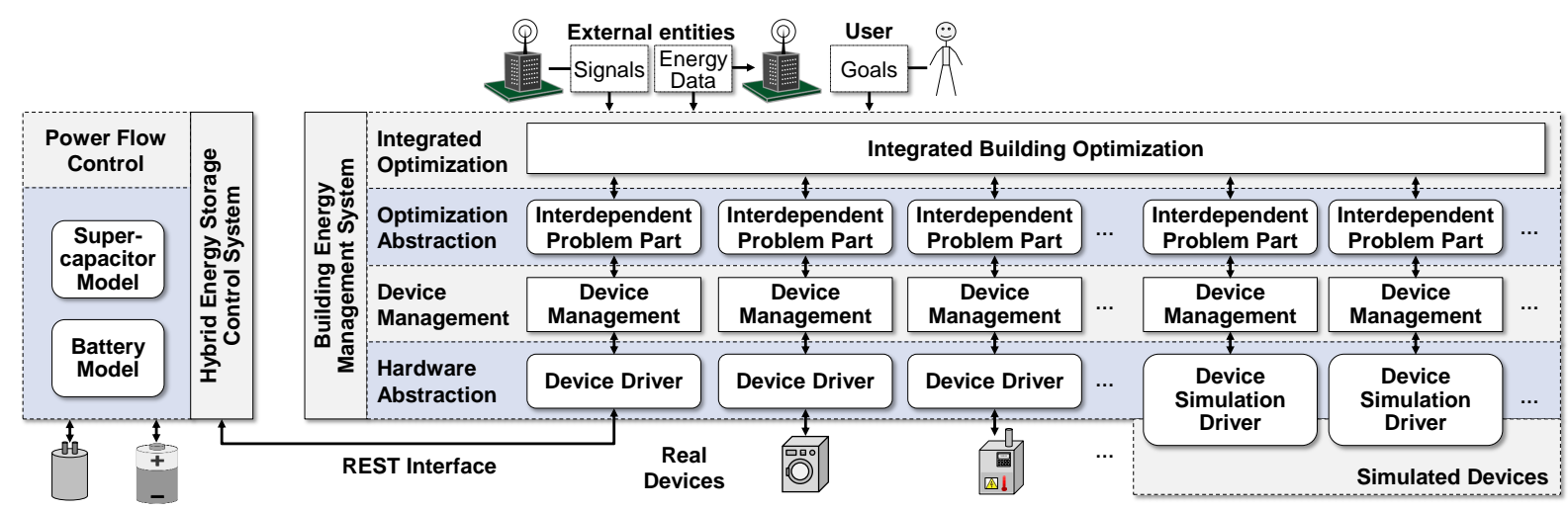

Figure 3: Architecture of the Organic Smart Home and the Hybrid Energy Storage Control System

\section{Energy Management and Simulation Software}

Energy management in smart buildings requires a BEMS that is able to optimize all devices in an integrated manner. The BEMS used in this paper is based on the Organic Smart Home ${ }^{2}$ (OSH) [10], it integrates additional software components that enable HIL scenarios and will interact with the HESCOS to fullfill the tasks demanded in Sect. II.

\section{A. Energy Management and Simulation System}

The ESHL is managed and optimized by the OSH, which has already been used in various smart building scenarios, such as a residential building comprising DG and electric vehicles [13] as well as a commercial building comprising a trigeneration system [14]. The OSH is used in both, real-world energy management in productive systems, such as the ESHL at KIT, and simulations of smart buildings, such as scenarios comprising buildings having hybrid home appliances [11] or stationary battery storage systems [15].

Figure 3 presents an overview of the general system architecture of the OSH, which is described in more detail in [10], [14], and the Hybrid Energy Storage Control System (see Sect. IV-B). The architecture comprises two major layers, which manage, optimize, and control the devices: the Device Management layer and the Integrated Optimization layer. These major layers utilize two auxiliary layers, a Hardware Abstraction layer to generalize devices and an Optimization Abstraction layer for their optimization. The integrated building optimization respects signals by external entities and optimizes the building's energy flows with respect to the user's goals, such as the minimization of total energy costs or the maximization of the self-consumption rate. The optimization in the OSH uses a Genetic Algorithm, i. e., a meta-heuristic, and an abstract representations of the devices in so-called Interdependent Problem Parts [14]. These abstract representations enable the optimization not only of operation times of appliances, ESS, and DG systems but also of other parameters, such as the parameters of control systems [15].

The abstraction of systems by the Hardware Abstraction enables to use the same implementations of the device

\footnotetext{
${ }^{2}$ http://www.organicsmarthome.com
}

management and optimization layers in both simulations and real environments. In simulations, Device Simulation Drivers replicate the behavior of the real devices. In productive realworld environments, Device Drivers realize the connection with real devices. The combination of both types enables HIL simulations. Furthermore, a message queue enables modular connections between the BEMS and ancillary services within the ESHL, like the occupancy prediction.

\section{B. Hybrid Energy Storage Control System}

The HESCOS is divided into two main parts: control of the grid interface and control of the hybrid storage.

Regarding the hybrid storage a power flow control will be implemented which allows that high peak power demands are handled by the EDLC storage, whereas for long term power delivery the batteries are used. For this purpose mainly two different approaches were presented in literature for hybrid storage systems in general: Considering the storage as an integrated system a multivariable control algorithm could be implemented, allowing high power quality and high control dynamics well suited for ancillary services [16], [17]. On the other hand also a control scheme like the well known droop control for DC-grids could be implemented. Here only little communication between battery and EDLC charge control is necessary, enabling a high flexibility for future extensions of the system, regarding for example an extension of storage capacity or additional power sources [18], [19].

The grid interface is realized with a predictive current controller. It allows a high quality current injection even under adverse conditions, enabling the demanded ancillary services (see Sect. II) like voltage support by reactive power, compensation of harmonics or increase of short circuit power.

All control algorithms are implemented and simulated in Matlab/Simulink. Using its code generation feature it is possible to build $\mathrm{C}$ code for a real-time control system consisting of a digital signal processor, so a fast cycle time from simulation to implementation on the real hardware is made possible. The real-time control system is capable to calculate the grid current control algorithm within $80 \mu$ s so even the control of the storage system could be implemented on the same processor, while enabling a switching frequency of $8 \mathrm{kHz}$ for the converter. This 


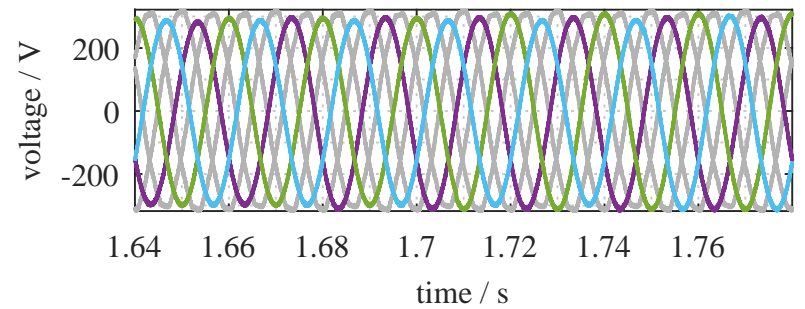

(a) Adaption of the phase voltages

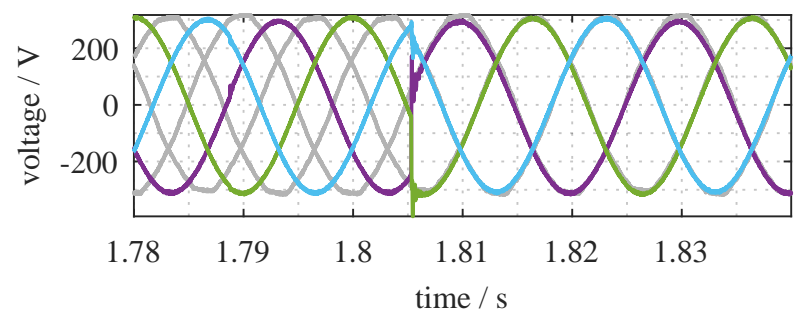

(b) Adaption of the phase angles

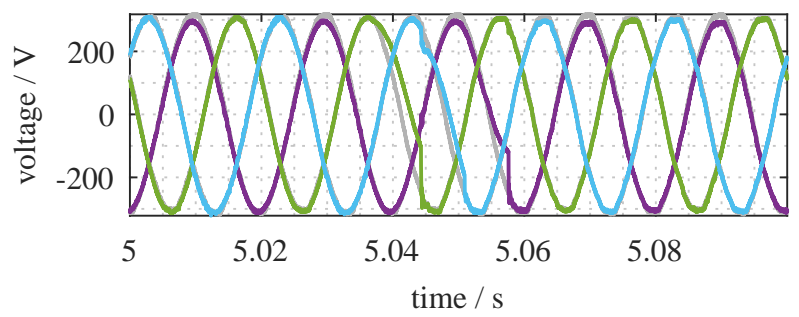

(c) Switch to stiff grid

Figure 4: Switching from emulated to stiff grid. The generated voltages of the 4-quadrant amplifier are depicted in Color. In Grey the voltages of the stiff grid are shown.

ensures a high flexibility regarding the selection of control schemes mentioned above.

The ESS real-time system will be connected and integrated into the OSH and its optimization via a REST-interface: The parameters of the ESS's control strategy are optimized within certain limits as described in [15].

\section{First Results}

In a first evaluation the ESHL has been operated in a 4quadrant-amplifier-driven PHIL simulation representing the connection to a weak grid connection point and evaluated the switching progress between stiff and artificial mains. The ESHL is assumed to be a solitary building connected via a $1000 \mathrm{~m}$ long four wire underground cable (NAYY 4x50 SE) with an impedance of $R=0.642 \Omega$ and a reactance of $X=$ $0.083 \Omega$. The 4-quadrant amplifier has been set to the given impedance and the voltages and currents at the household's connection point have been monitored. The measurements have been carried out on a spring day in the late afternoon, the PV inverter was disabled during the measurement period. The attendance of inhabitants within the building has been simulated by using different devices and appliances of the lab (L1: hood; L2: dishwasher, freezer; L3: kettle, hob; 3-phase: microCHP).

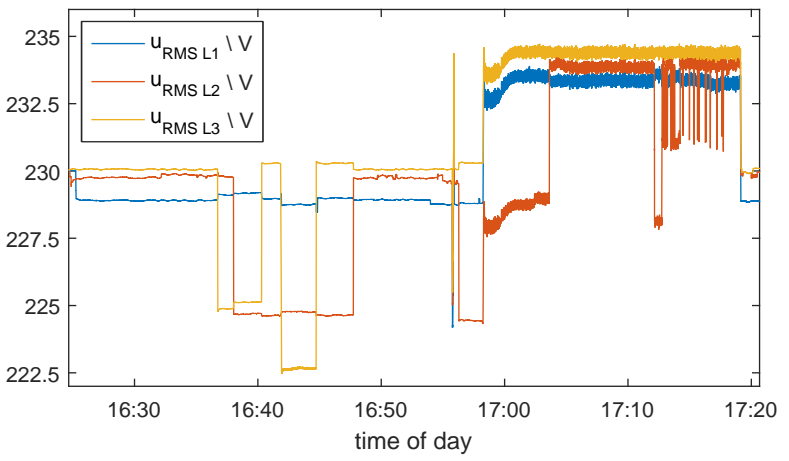

(a) U: 3-phase voltages

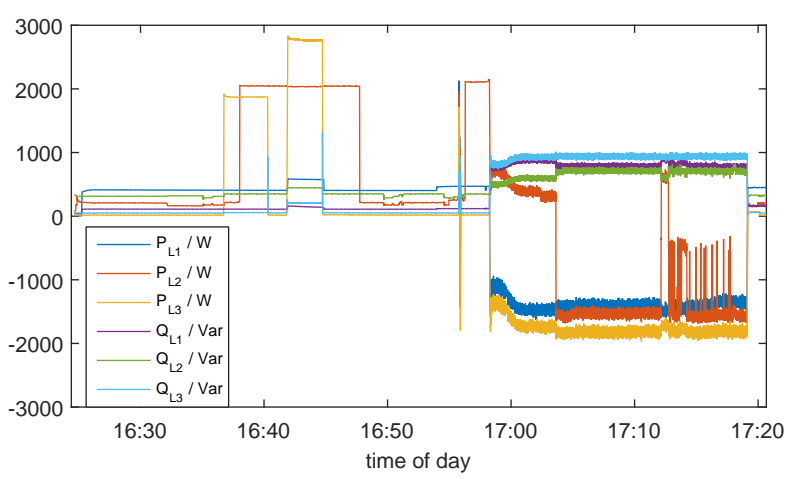

(b) P, Q: active power. reactive power

Figure 5: P, Q, U: 3-phase measurement at the ESHL grid connection point during 4-quadrant amplifier operation

\section{A. Automatic Switching}

To limit the inrush-currents during switching, it has been divided into three parts: Firstly, adaption of the grid voltages from stiff to artificial mains (Fig. 4a). Secondly, synchronization of the emulated grid phase to the phase of the stiff grid (Fig. 4b). Finally, switching action of the switching box (Fig. 4c). The shown switching action results in a seamless transfer between emulated and stiff grid, so operation of the ESHL is maintained during the transitions. This allows fast emulation and test of different grid conditions (see Sect. V-B) because a longer shutdown for a manual transition to the emulated grid is not necessary.

\section{B. Intelligent Building in a Weak Distribution Grid}

In Fig. 5, the 3-phase (L1, L2, L3) measurements of the voltages $U_{\text {RMS }}$ (a) and active as well as reactive powers (b) are shown. There is a strong anti-correlation between the phase voltage and the corresponding active power, whereas the reactive power has less effect on the voltage. This behavior is typical for LV grids due to the high $\mathrm{R} / \mathrm{X}$-ratio of typically used cables. Thus, the active power balance has a greater impact on the bus voltages than the reactive power.

\section{CONCLUSION AND OUTLOOK}

Within this paper an approach for evaluation of smart grid technologies for LV grids has been presented. 
DG and emerging storage systems are causing stability, power quality, and grid congestion issues. The related decrease in short circuit power requests also for new technologies. To address these challenges, all categories of equipment-DG, flexible loads, energy storage, and power storage - have been integrated into a lab - a residential building — to build an evaluation platform for innovative smart grid hard- and software. The approach utilizes a combination of conventional simulations, small signal feedback HIL simulations and large signal feedback PHIL simulations for the evaluation of novel technologies. As first results of the ongoing research, the switching and operation of the labs power supply between a stiff and an artificial mains network provided by a 4-quadrant amplifier as well as the operation of the lab on a simulated weak grid connection point have been presented. Future work will be on closing the control loops of the presented HIL and PHIL approaches for energy management and ancillary services as well as on research on control strategies for autonomous and signal-driven smart homes.

\section{REFERENCES}

[1] B. Becker, I. Mauser, H. Schmeck, S. Hubschneider, and T. Leibfried, "Smart Grid Services Provided by Building Energy Management Systems," in 2015 Proceedings of the IEEE PES Conference on Innovative SMART GRID Technologies (ISGT-LA). IEEE, 2015, pp. 851-856.

[2] G. Lammert, T. Hes, M. Schmidt, P. Schegner, and M. Braun, "Dynamic grid support in low voltage grids - fault ride-through and reactive power/voltage support during grid disturbances," in Power Systems Computation Conference. IEEE, Aug 2014.

[3] S. Kochanneck, C. Hirsch, I. Mauser, H. Schmeck, and M. Schröder, "Bottom-Up Simulation of Suburban Power Grids," in ETG Congress 2015: Die Energiewende - Blueprint for the new energy age. VDE VERLAG, 2015.

[4] S. Kochanneck, H. Schmeck, I. Mauser, and B. Becker, "Response of smart residential buildings with energy management systems to price deviations," in 2015 IEEE Innovative Smart Grid Technologies - Asia, 2015.

[5] R. Isermann, J. Schaffnit, and S. Sinsel, "Hardware-in-the-loop simulation for the design and testing of engine-control systems," Control Engineering Practice, vol. 7, no. 5, pp. 643-653, 1999.
[6] A. Bouscayrol, "Different types of hardware-in-the-loop simulation for electric drives," in IEEE International Symposium on Industrial Electronics (ISIE), 2008, pp. 2146-2151.

[7] B. Becker, F. Kern, M. Loesch, I. Mauser, and H. Schmeck, "Building energy management in the fzi house of living labs," in Energy Informatics, Chapter 9, ser. Lecture Notes in Computer Science. Springer, 2015, vol. 9424 , pp. $95-112$.

[8] C. Düpmeier, K.-U. Stucky, R. Mikut, and V. Hagenmeyer, "A Concept for the Control, Monitoring and Visualization Center in Energy Lab 2.0," in Energy Informatics. Springer, 2015, pp. 83-94.

[9] S. Rohjans, S. Lehnhoff, and M. Büscher, "SESA-Lab: Gesamtsystemische Smart Grid Simulationen Generischer Automatisierungsarchitekturen," in VDE-Kongress 2014. VDE VERLAG, 2014.

[10] F. Allerding, "Organic Smart Home - Energiemanagement für Intelligente Gebäude,” Ph.D. dissertation, Karlsruhe Institute of Technology (KIT), Germany, 2013.

[11] I. Mauser, H. Schmeck, and U. Schaumann, "Optimization of Hybrid Appliances in Future Households," in ETG Congress 2015: Die Energiewende - Blueprint for the new energy age. VDE VERLAG, 2015.

[12] H. Maass, H. K. Cakmak, W. Suess, A. Quinte, W. Jakob, K. U. Stucky, and U. Kuehnapfel, "Introducing the electrical data recorder as a new capturing device for power grid analysis," in Applied Measurements for Power Systems (AMPS), IEEE Int. Workshop on, Sept 2012, pp. 1-6.

[13] M. Mültin, F. Allerding, and H. Schmeck, "Integration of electric vehicles in smart homes - an ICT-based solution for V2G scenarios," in 2012 IEEE PES Innovative Smart Grid Technologies (ISGT), 2012, Inproceedings.

[14] I. Mauser, J. Müller, F. Allerding, and H. Schmeck, "Adaptive Building Energy Management with Multiple Commodities and Flexible Evolutionary Optimization," Renewable Energy, vol. 87, Part 2, pp. 911-921, 2016.

[15] J. Müller, M. März, I. Mauser, and H. Schmeck, "Optimization of Operation and Control Strategies for Battery Energy Storage Systems by Evolutionary Algorithms," in Applications of Evolutionary Computation. Springer International Publishing, 2016.

[16] C. Jin, N. Lu, S. Lu, Y. Makarov, and R. A. Dougal, "Coordinated control algorithm for hybrid energy storage systems," in IEEE Power and Energy Society General Meeting, Jul 2011.

[17] F. Yang, B. Sheng, and Y. Fu, "Energy management for fuel cellsupercapacitor hybrid system using passivity-based controller with multiequilibrium states," in IECON 2015 - 41st Annual Conference of the IEEE Industrial Electronics Society, Nov 2015.

[18] T. Dragicevic, J. M. Guerrero, J. C. Vasquez, and D. Skrlec, "Supervisory control of an adaptive-droop regulated DC microgrid with battery management capability," in Transactions on Power Electronics, vol. 2-29. IEEE, Feb 2014, pp. 695-706.

[19] G. Zhang, X. Tang, and Z. Qi, "Research on battery supercapacitor hybrid storage and its application in microgrid," in 2010 Asia-Pacific Power and Energy Engineering Conference. IEEE, 2010. 